\title{
Use of Growth-Rate/Temperature-Gradient Charts for Defect Engineering in Crystal Growth from the Melt
}

\author{
Thierry Duffar
}

SIMAP, Grenoble INP, CNRS, University Grenoble Alpes, 38000 Grenoble, France; thierry.duffar@grenoble-inp.fr

Received: 27 August 2020; Accepted: 1 October 2020; Published: 8 October 2020

\begin{abstract}
As the requirements in terms of crystal defect/quality and production yield are generally contradictory, it is necessary to develop methods in order to find the best compromise for the growth conditions of a given crystal. Simple growth-rate/temperature-gradient charts are a possible tool in this respect. After the recall of the classical analytical equations useful for describing the process and defect engineering, a simple pedagogic case explains the building and use of such charts. The more complex application to the directional casting of photovoltaic Si necessitated the development of new physical models for twinning and equiaxed growth. This allowed plotting charts that proved useful for industrial applications. The conclusions discuss the drawbacks and advantages of the method. It finally proves to be a pedagogic tool for teaching crystal growth engineering.
\end{abstract}

Keywords: crystal growth from the melt; process engineering; defects; diagrams; photovoltaic Si directional solidification; pedagogy

\section{Introduction}

The industrial production of single crystals with controlled quality and reasonable yield is always a matter of compromise. For instance, the growth rate should be as large as possible in order to increase productivity; however, a too large growth rate is likely to be the source of defects such as large bubble incorporation or interface destabilization. The interface can be stabilized under large temperature gradients; however, they produce high thermo-elastic stresses, hence dislocations and even cracks. Finding the best compromise may become quite difficult when several contradictory requirements should be fulfilled simultaneously. Most of the time, this has been solved, in practical cases, by experimental trial and error.

The use of quality-process charts, or diagrams, is a helpful tool for finding the best compromise. We began to develop this approach at the very beginning of the 1990s, when our laboratory was thinking about the development of a Bridgman furnace for the growth of GaAs single crystals of various qualities. This approach is not strictly original, as in the same period, the use of diagrams was under development by metallurgists [1]. Their problem was to find which process parameters could produce a metallic alloy with a given microstructure (in terms of dendrites, eutectics, their size and proportions, etc.). Diagrams, such as presented in Figure 1, were produced for various alloy classes. This has been subsequently developed with connections to thermodynamic and kinetic databases [2]. To quote Kurz's point of view, "A combined approach of microstructure mapping with process mapping is developed which allows a presentation of processing windows leading to specific microstructures for given alloy compositions and processing conditions".

A similar approach is developed in the present paper in the field of crystal growth. It is restricted to growth from the melt but can be used for other technologies. It is based on the principle that, whatever the grown crystal (semiconductor, oxide, etc.) or the production process (Czochralski, Bridgman, etc.) the basic physical phenomena are the same and only physical parameters change, so the approach is common and useful in any case. While these phenomena have been studied for more than fifty years, they are commonly used alone. The idea is to consider them simultaneously for optimization purposes. 


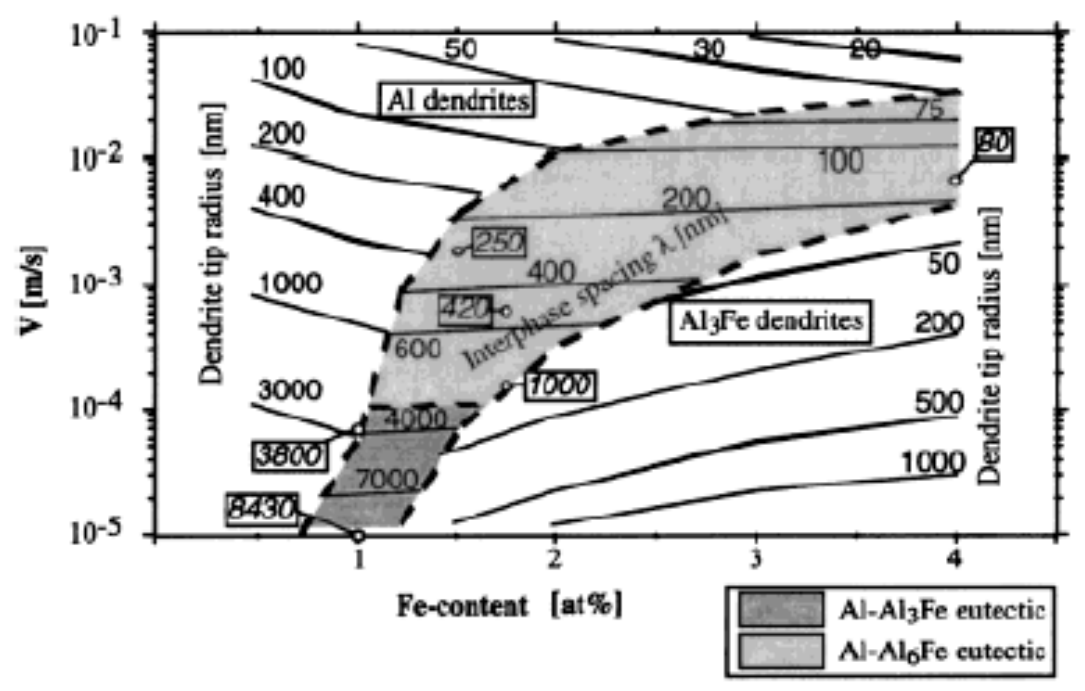

Figure 1. Example of a diagram as used in the metallurgy of Al-Fe alloys. In this Fe concentration/growthrate diagram, different areas are defined, where various microstructures, such as dendrites or eutectics, develop. Numbered lines give the characteristic sizes of the microstructure. From [2].

Another interest in such diagrams concerns education. When, later on, I became a professor in the materials science department of my university, I developed lectures on crystal growth engineering. Such charts are still, today, the basis of these lectures, because they allow one to introduce all the important aspects of crystal growth engineering, in terms of defects and processes, and, in this way, appear to be an excellent pedagogic tool.

Rather intriguingly, considering that it was introduced some 30 years ago and is commonly used in lectures, to my knowledge, this approach has never been published to date in the field of crystal growth. The purpose of this article is to present how these diagrams are constructed and used, with the hope that it could be useful for a wider engineering or teaching audience. The basic equations used in order to plot such diagrams are, of course, well known; they are simply recalled in the next section. The third part provides an example of the building of a diagram based on these classical equations, for the design of a Czochralski setup. The fourth paragraph shows that the approach can be further developed, for instance, in the case of the grain structure of photovoltaic silicon. The conclusions focus on possible further developments of the use of charts in crystal growth.

\section{Classical Basic Equations Involved in Crystal Growth from the Melt}

Figure 2 represents a schematic crystal with the defects that could be inherited from the process conditions. While the equilibrium state of the crystal can be computed through thermodynamic analysis, defects are due to kinetic, non-equilibrium phenomena, namely:

- Heat transfer, modelled by Fourier's equation, and, possibly, radiative exchanges. Temperature controls chemical reactions, i.e., with crucibles or casing. Temperature gradients generate thermoelastic stresses in the solid and convection in the melt. Most importantly, the movement of the solidliquid $(\mathrm{S} / \mathrm{L}$ ) interface is the movement of an isothermal surface, at least for pure-enough materials.

- Mass transfer modelled by Fick's laws. It controls chemical homogeneity through species transport in the melt and point defect distribution in the crystal.

- Hydrodynamics, modelled by Navier-Stokes equations. Liquid and gas movements impact heat and mass transfer, which, in turn, generate fluid flows.

- Thermo-mechanical stresses modelled by Hooke's law. They are likely to produce dislocations, sub-grain boundaries and, in the worst case, cracks. 
- Interfaces, first of all the S/L boundary, control the incorporation of constituents, dopants and impurities and, when spurious nucleation occurs, the grain or twin structure. Fluid-fluid interfaces affect the size and shape of the crystal through the fluid menisci used in many capillarity-controlled processes.

All these phenomena are modelled by second-order differential equations that, in the general case, can be solved today with well-developed numerical simulation software. However, the optimization of a process in this way would require a tremendous amount of calculations if no approximate initial solution of the problem were available.

Indeed, an older approach consisted of deriving analytical solutions of these equations in idealized cases representing common crystal growth situations. Such developments were made some fifty years ago or more, but, unfortunately, they are underused today, while they are a perfect way to find a good approximation of the optimized growth conditions.

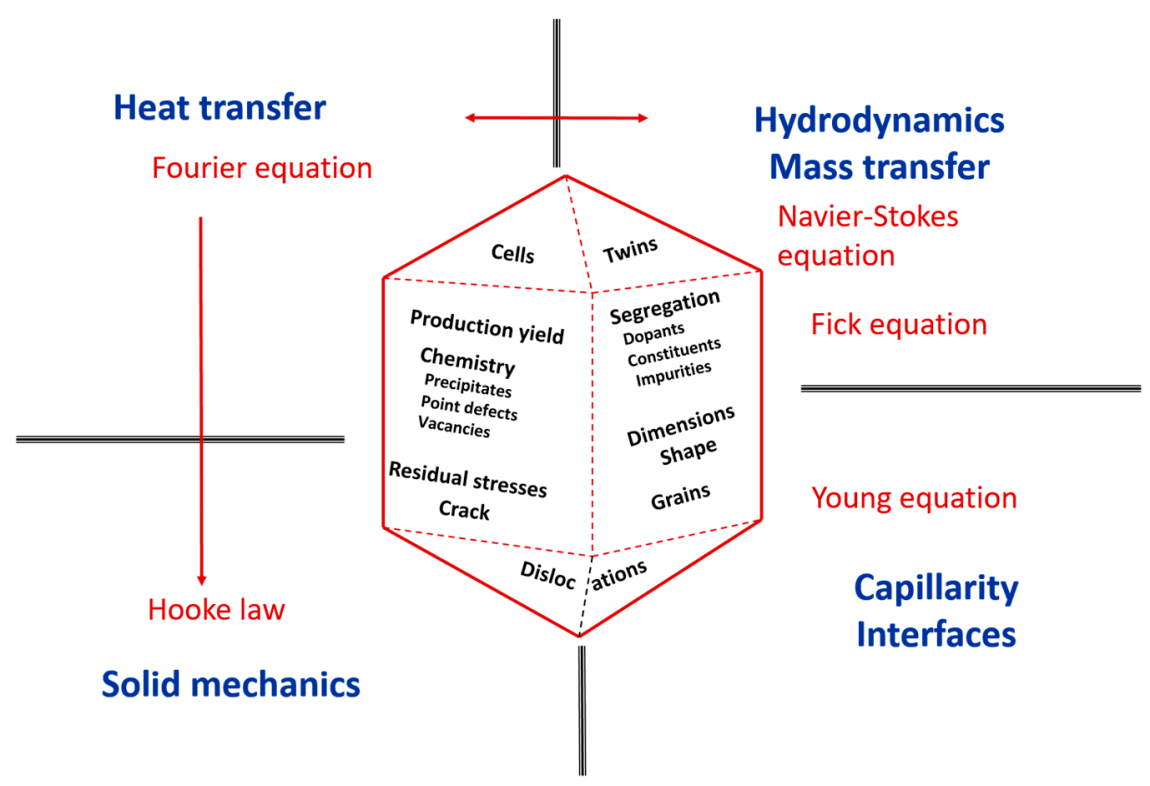

Figure 2. Fundamental physical phenomena impacting the occurrence of defects in a crystal.

Our goal is not to provide an exhaustive list of all possible equations derived for the analysis of crystal growth from the melt, nor to recall their demonstrations. Generally, more precise but complex equations can also be found in the literature. Hence, here is rather an example list of some equations, restricted to those used in this paper, which can be considered as "rules of thumb" but appear, from our experience, to be useful and reliable in practical cases.

\subsection{Equations Related to the Process}

- Heat balance. This is the most important equation in the field of solidification and is known as the "Stefan condition", from the name of J. Stefan, who, in the 1880s, derived the sea frozen thickness as a function of the number of freezing days [3]. It is the heat balance at the $\mathrm{S} / \mathrm{L}$ interface, which simply states that the heat flowing through the solid is the sum of the heat coming from the liquid and the latent heat released by the solidification of the material:

$$
k_{L} \nabla T_{L}+V \Delta H_{m}=k_{S} \nabla T_{S} .
$$

- Cooling rate. It is useful for those processes where solidification is driven by the cooling of a heater, furnace, etc. It is related to other process parameters through:

$$
\dot{T}=\nabla T_{S} V .
$$


- Constant growth rate. While never clearly demonstrated theoretically, it is common experience among crystal growers that it is preferable to keep the growth rate constant in order to obtain a crystal of good structural quality.

- Other process-related equations include temperature stability criteria in the case of the crystal shape being controlled by capillarity [4]. Additionally, in a given geometry, the temperature gradient is often constrained by a limit temperature due to evaporation, chemical reactions with the crucible or the atmosphere.

\subsection{Equations Related to the Crystal Defects and Structure}

- Interface stability. When a solute (dopant, impurity, additional constituent, etc.) is rejected into the melt, Chalmers and co-workers [5] demonstrated that there is a chemically enriched layer at the interface leading to liquid undercooling. If the temperature gradient in the liquid is not large enough, this undercooled liquid tends to solidify so that the solid-liquid interface becomes unstable. The interface remains stable and then can produce a single crystal, when:

$$
\frac{\nabla T_{L}}{V}>\frac{-m_{L} C_{0}(1-k)}{k D_{L}} .
$$

This behavior is characteristic of multicomponent melts and strongly determines the quality of such alloyed crystals.

- Chemical segregation. Sheil [6] derived the equation of solute segregation in the case of a finite melt continuously homogenized by mixing. This is, in practice, applicable to most crystal growth processes. It is generally written in the form:

$$
C_{S}(f)=C_{0} k(1-f)^{(k-1)}
$$

Other equations are applicable for some specific processes. In the practical case, $k$ is replaced by an "effective" segregation coefficient, $k_{e f f}$, which takes into account the growth rate and the degree of mixing of the melt.

- Stresses and dislocations. Based on estimations of temperature gradients and stresses by Billig [7], the problem of dislocation generation under stresses experienced by the crystal during its growth was actively studied in the 1970s [8]. The accommodation of the deformation exceeding the yield point by an adequate number of dislocations, each providing its Burgers vector, provides a rough estimate of the resulting dislocation density:

$$
N_{D}=\frac{\alpha}{24 b} \nabla T_{S}-\frac{\sigma_{C R S S}}{E R b},
$$

from which it follows that a crystal without dislocations can be obtained if:

$$
\nabla T_{S}<\frac{24 \sigma_{C R S S}}{E R \alpha} .
$$

There are many other such equations dealing, for example, with point defect generation in semiconductors, eutectic spacing, bubble or particle engulfment, the occurrence of crystal cracks and other problems. Looking at this collection of equations, it appears, clearly, that the three main parameters controlling the quality of the crystal, through the process, are:

- $\quad$ The growth rate, $V$, imposed by the pulling shaft or by furnace cooling.

- One of the temperature gradients in the liquid or solid, $\nabla T_{L}$ or $\nabla T_{S}$, as they are both linked by $V$ in Equation (1). The importance of this parameter is largely underestimated: very few papers on experimental crystal growth provide gradient values, which makes difficult the analysis of the described results. Additionally, they are generally unknown in industrial processes. This is 
certainly due to the fact that it is very difficult to measure the temperature field in the crystal or in the liquid, which generally requires mockup materials, when available. Alternatively, a numerical simulation of heat transfer in the process should be developed.

- The chemical composition of the material, $C_{0}$, which is not a free parameter, as it is imposed by the crystal application.

Other important parameters in crystal growth are related to process chemistry, such as the raw material impurity level, growth atmosphere or crucible material. They can be studied independently by thermodynamic calculations, based on the expected maximum temperature.

Finally, it can be concluded that, for a given crystal with an expected concentration and radius, all phenomena related to the crystal growth quality and process can be represented in a $V-\nabla T$ diagram.

\section{Case Study: Growth of GaSb in a Czochralski Setup}

In order to explain the building and use of process-defect charts, we will develop a simple example, namely, the final exercise of our crystal growth engineering lectures. It states the following: "Find optimal conditions for the Czochralski growth of dislocation-free, Te doped (250 ppm), GaSb single crystals ( $\varnothing=$ $40 \mathrm{~mm}, L=30 \mathrm{~cm})^{\prime \prime}$. The necessary physical properties are given in Table 1 .

Table 1. Physical properties of Te:GaSb.

\begin{tabular}{cc}
\hline$\alpha$ & $7 \times 10^{-6} \mathrm{~K}^{-1}$ \\
\hline$E$ & $8.7 \times 10^{4} \mathrm{MPa}$ \\
\hline$\sigma_{C R S S}$ & $0.5 \mathrm{MPa}$ \\
\hline$k_{L}$ & $10.2 \mathrm{~W} \cdot \mathrm{m}^{-1} \cdot \mathrm{K}^{-1}$ \\
\hline$k_{S}$ & $6.4 \mathrm{~W} \cdot \mathrm{m}^{-1} \cdot \mathrm{K}^{-1}$ \\
\hline$\Delta H$ & $0.2 \times 10^{6} \mathrm{~J} \cdot \mathrm{kg}^{-1}$ \\
\hline$\rho$ & $6 \times 10^{3} \mathrm{~kg} \cdot \mathrm{m}^{-3}$ \\
\hline$k$ & 0.3 \\
\hline$D_{L}$ & $0.8 \times 10^{-8} \mathrm{~m}^{2} \cdot \mathrm{s}^{-1}$ \\
\hline$m_{L}$ & $-2 \times 10^{-4} \mathrm{~K} \cdot(\mathrm{ppm})^{-1}$ \\
\hline
\end{tabular}

As a growth-rate/temperature-gradient chart is to be built, the first step is to find appropriate scales for these two parameters. For this, it is necessary to find, in the applicable equations, which one could provide a numerical value for the growth rate or the temperature gradient. In the case of interest, a first requirement concerns the absence of dislocations. Using Equation (6) provides the maximal temperature gradient in the solid:

$$
\nabla T_{S} \leq 10 \mathrm{~K} \cdot \mathrm{cm}^{-1}
$$

Using Equation (1) allows plotting the green line in a $V-\nabla T$ diagram as presented in Figure 3. This, already, defines a triangular region in the diagram showing acceptable growth conditions. However, another requirement is to be taken into account. As the crystal is doped, the stability of the S/L interface should also be secured. The criterion is provided by Equation (3). It provides the blue line in the diagram. Finally, the allowed domain for the growth of such crystals is the red-hatched triangle.

The last step is to determine the operating point for the process. Maximizing the production yield involves selecting the highest possible growth rate; therefore, taking into account some safety margins, the red star represents the best growth conditions. The lower axis provides the growth duration for a $30 \mathrm{~cm}$ crystal, which shows that a complete pulling lasts 1.5 days. 


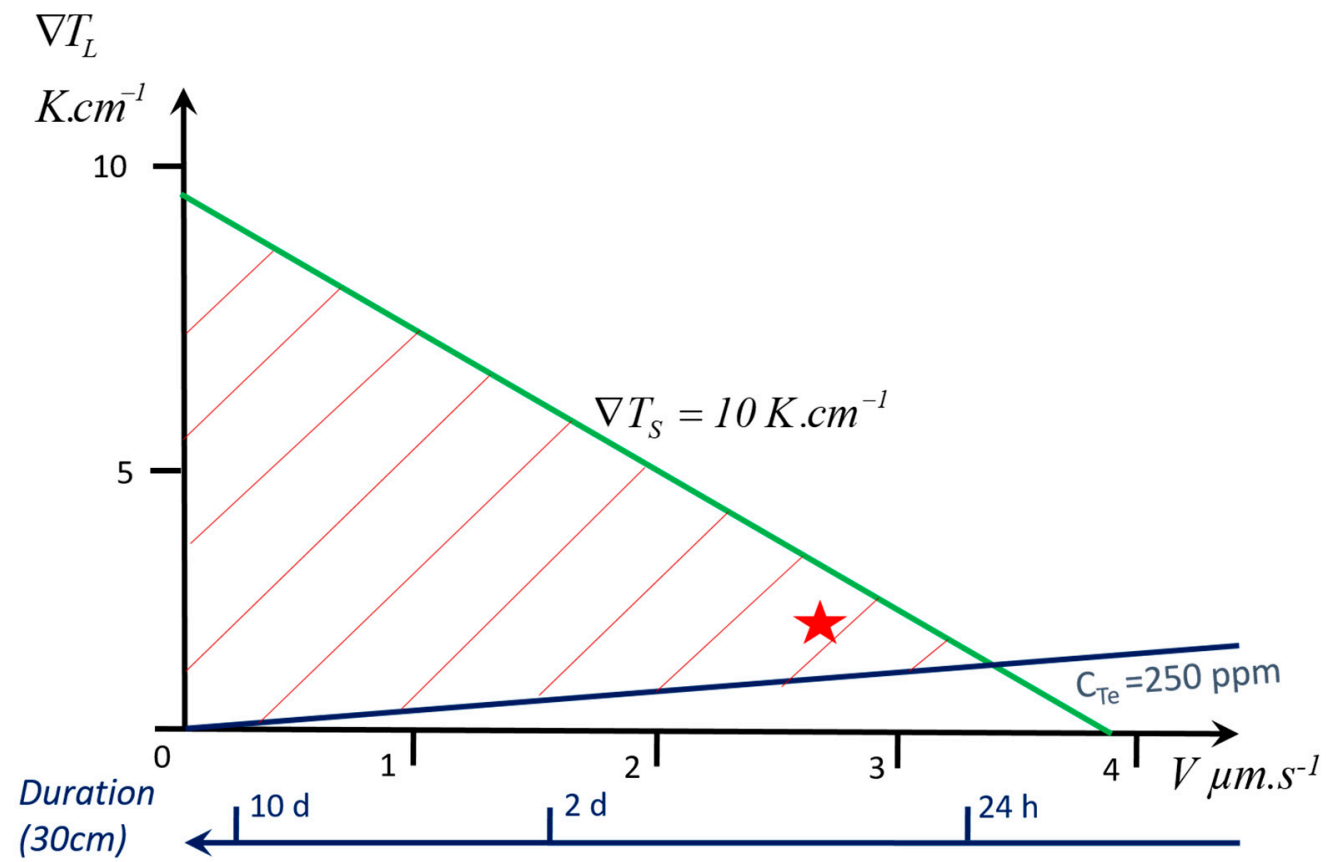

Figure 3. Diagram obtained for the optimization of the growth of Te:GaSb single crystals.

A more reasonable treatment would take into account the chemical segregation of Te, Equation (4), which would lead to modifying the diagram as shown in Figure 4. Then, the amount of useful crystal, considering the limit of dopant concentration, should be used in order to find the best growth conditions: this is likely to reduce the pulling rate and increase the growth duration. It also increases the temperature gradient and then the heat flow and the overall equipment power. Of course, other requirements, considering, for example, the crystal shape stability or point defect level, could also provide lines to be added on the graph.

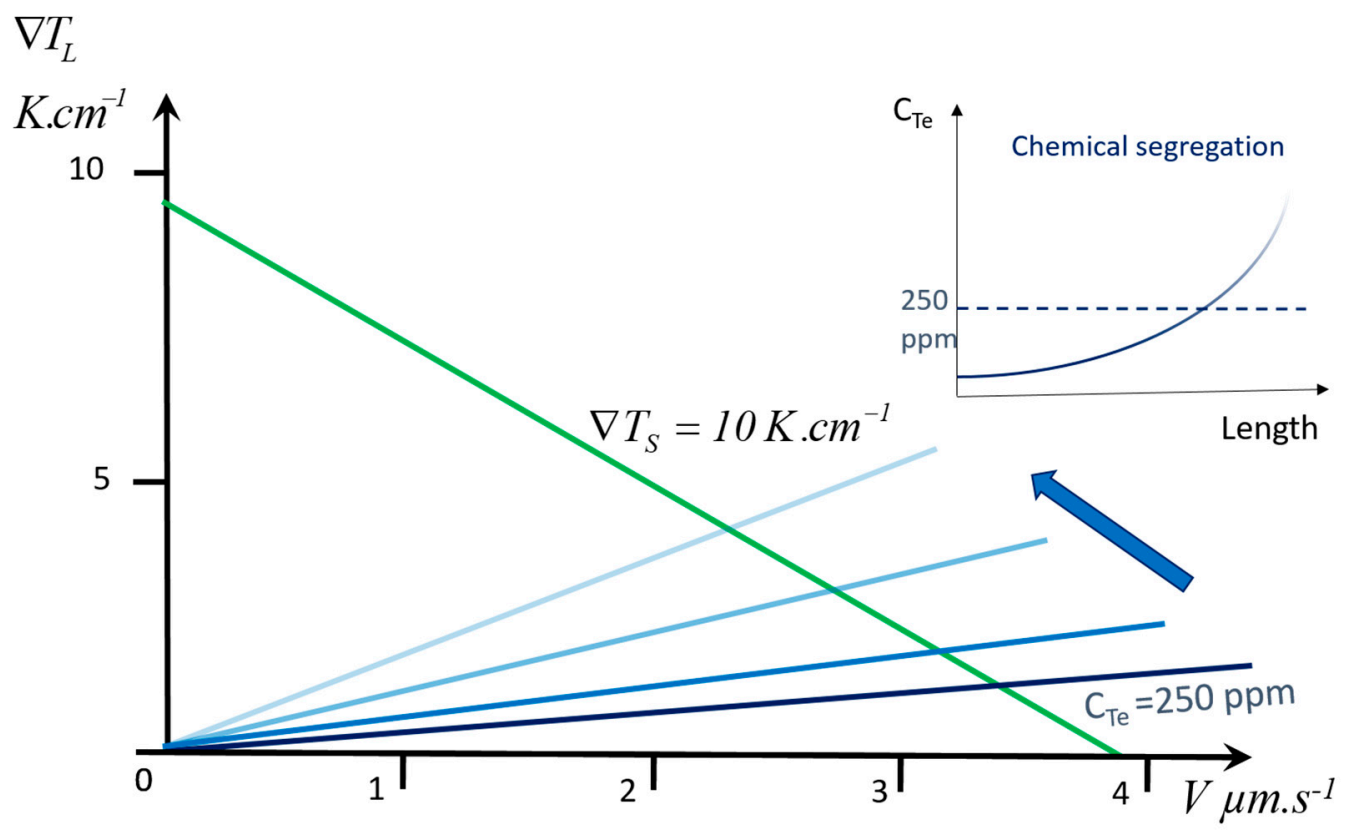

Figure 4. Diagram modified in order to take into account the chemical segregation of tellurium. Its increase with length changes the interface stability criterion, following the arrow. 
From the obtained growth conditions, it is possible to begin thinking about the setup design, in terms of the pulling requirements, heat source and insulation to provide the requested temperature gradient. Of course, this is a crude and simplified analysis of the growth process. However, it should be noted that building such a diagram takes a very short time (the longest is to find the appropriate physical properties) and represents virtually no cost. It can be improved in two directions: the use of more precise, but more complex, physical descriptions and the development, from the obtained conditions, of a numerical simulation of the expected process and setup, in order to obtain a more precise and realistic design.

\section{Development of Chart for Grain Control in Photovoltaic Si Directional Solidification}

This second example is a summary of our activity in the field of the grain boundary structure in photovoltaic silicon ingots. This research was performed in close collaboration with industrial partners, and the objective was to find how the grain structure depends on the growth process parameters. Figure 5 shows the grain structure of a typical Si wafer used for the production of solar cells.

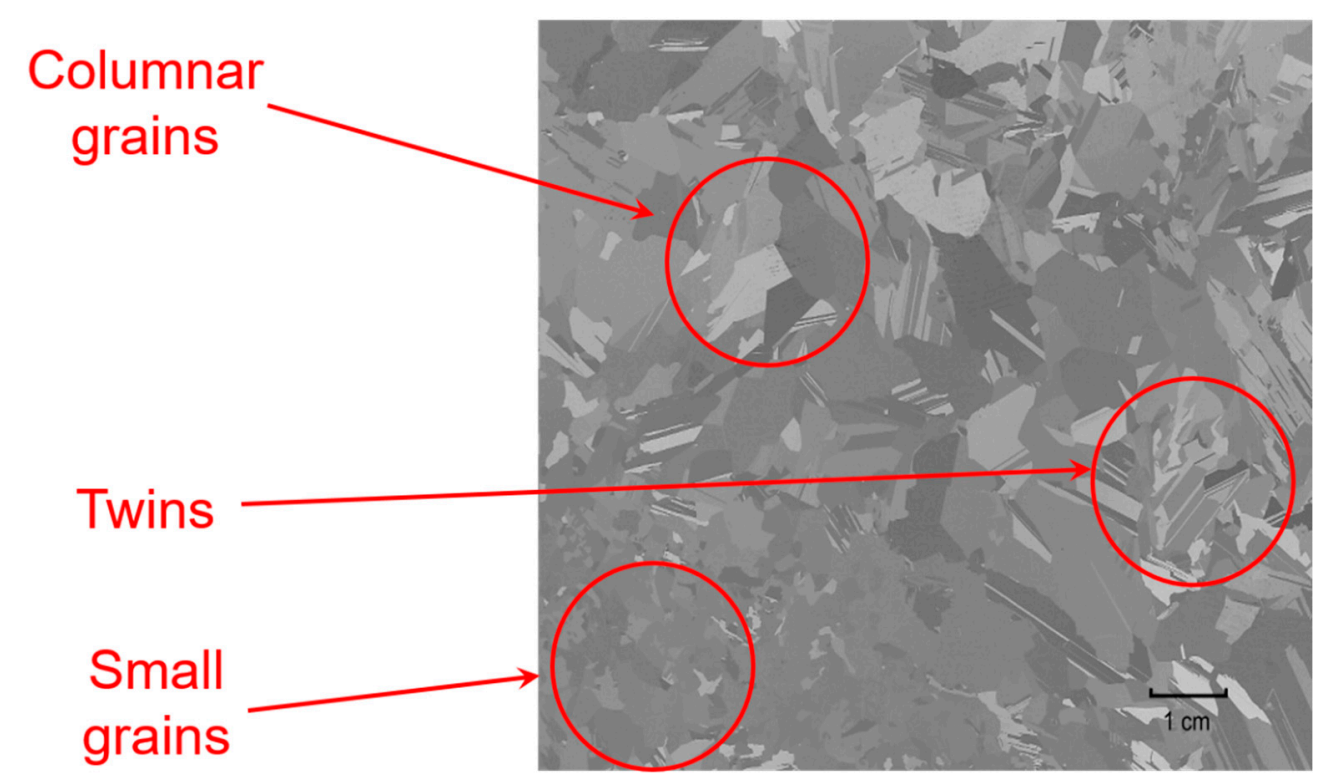

Figure 5. Grain structure of a Si solar cell wafer.

Several grain types appear:

- Large grains, called "columnar", as they grow perpendicularly to the S/L interface and extend along the ingot height.

- Some regions where small grains appear. They are called "equiaxed", as their shape is rather spherical. They generally occur in C-contaminated raw materials, at the top of the ingot, where the solute is likely to be segregated (see Equation (4)).

- $\quad$ Some grains are striped by parallel lines, which, in fact, are a sign of intense twinning.

No analytical model, like those presented in Part 2, was available in order to relate the grain structure to growth conditions, so it was necessary to propose and quantify theoretical explanations before establishing charts.

Many papers discuss the faceting capability of $<111>$ planes in $\mathrm{Si}[9]$ and the twinning occurring on such facets [10]. Therefore, the twinning problem was to find under which conditions the S/L interface is mainly rough or facetted. The undercooling of a facet is directly dependent on the growth rate: for $\mathrm{Si}$, the best available relationship has been provided in [11]. For a given growth rate, and therefore undercooling, the proportion of the facetted area on the $\mathrm{S} / \mathrm{L}$ interface depends directly on the temperature gradient, as shown schematically in Figure 6a-c [12]. 

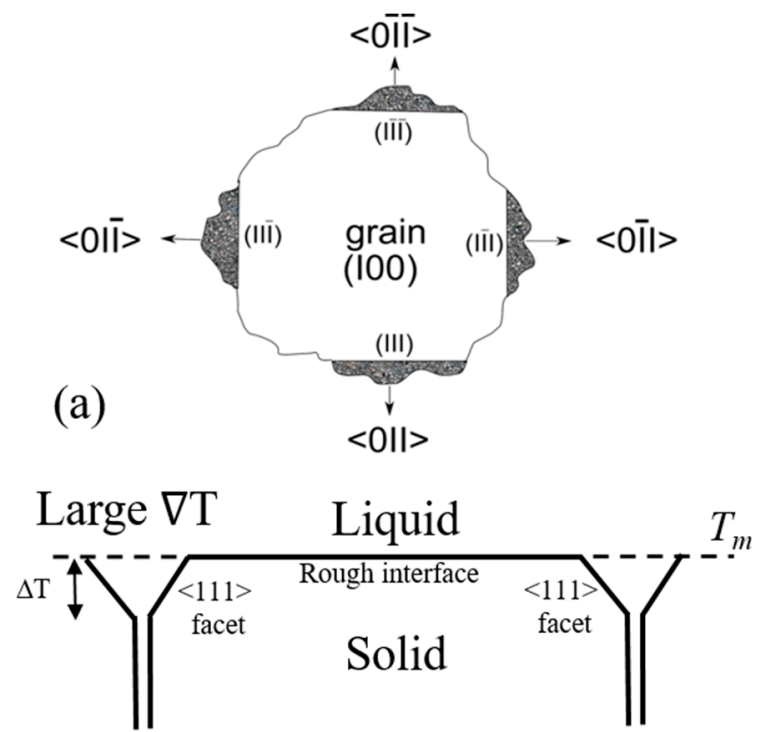

(b)

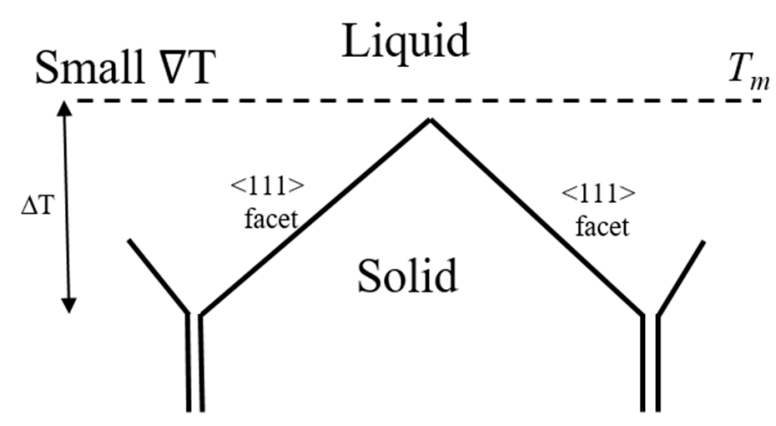

(c)

Figure 6. (a) A (100) grain at the solid-liquid (S/L) interface, seen from the liquid, showing four $<111>$ facets on its boundary. (b) Cut view of this grain, showing small facets and a large rough interface, under a large temperature gradient. (c) Cut view of the same grain with the same facet undercooling, showing that it is totally faceted under a small temperature gradient. Adapted from [12].

The blue line in Figure 7 shows the limit between a totally facetted and a rough S/L interface for Si in the case of grains $1 \mathrm{~cm}$ in size. In the facetted area, twinning is most probable, while it is very rare in the rough area.

The occurrence of small, equiaxed grains is more complex and needed several years of research. It finally can be summarized as follows $[13,14]$ :

- This occurs in the faceted zone of the diagram, where the mean temperature of the S/L interface is lower than the Si melting point (as can be seen in Figure 6c): there is an undercooled liquid layer in contact with the $\mathrm{S} / \mathrm{L}$ interface.

- $\quad$ The carbon is segregated ahead of the S/L interface and, when the solubility limit is reached, precipitates as $\mathrm{SiC}$.

- $\quad$ These SiC particles act as nucleation centers for the undercooled Si just above the S/L interface [15]. Therefore, equiaxed, facetted grains of Si appear and grow ahead of the main, columnar interface.

- There is competition between the equiaxed and the columnar grains, which decides the local equiaxed or columnar structure of the ingot. This was modelled in the same way as equiaxed growth in metallurgy [16], with the difference that both equiaxed and columnar grains are facetted in the case of $\mathrm{Si}$. 
These phenomena have been described with the appropriate known equations; the interested reader should refer to $[13,14]$ for a detailed description of the model.

Figure 7 is an original attempt to present, simultaneously, the results of the two approaches described above. The limit between columnar and equiaxed structures, for an amount of $C$ in the melt close to the solubility limit, is schematically shown as a red line in Figure 7. Therefore, there are three zones in this diagram. In the A zone conditions, the ingot is essentially constituted of columnar vertical grains with good photovoltaic properties. In the B zone, the columnar grains are facetted and then present twinning. It has been shown that a few successive twinning events quickly produce a structure of randomly oriented grains [17], leading to a decrease in PV properties. Finally, in the $\mathrm{C}$ zone, the structure is composed of tiny equiaxed grains, which provide low-quality cells and are likely to damage the slicing saws because of the hardness of the SiC precipitates. The growth conditions of the industrial process are represented by the red star in the diagram. It is located in the B region, explaining why twins are very frequent in the ingots. It is also close to the equiaxed zone, so equiaxed structures appear occasionally, when the local carbon concentration, or the local growth rate, increases accidentally. Finally, our advice has been to increase the vertical temperature gradient in the furnace, which is, however, limited by the occurrence of dislocations-Equation (5)—or cracks, not discussed here.

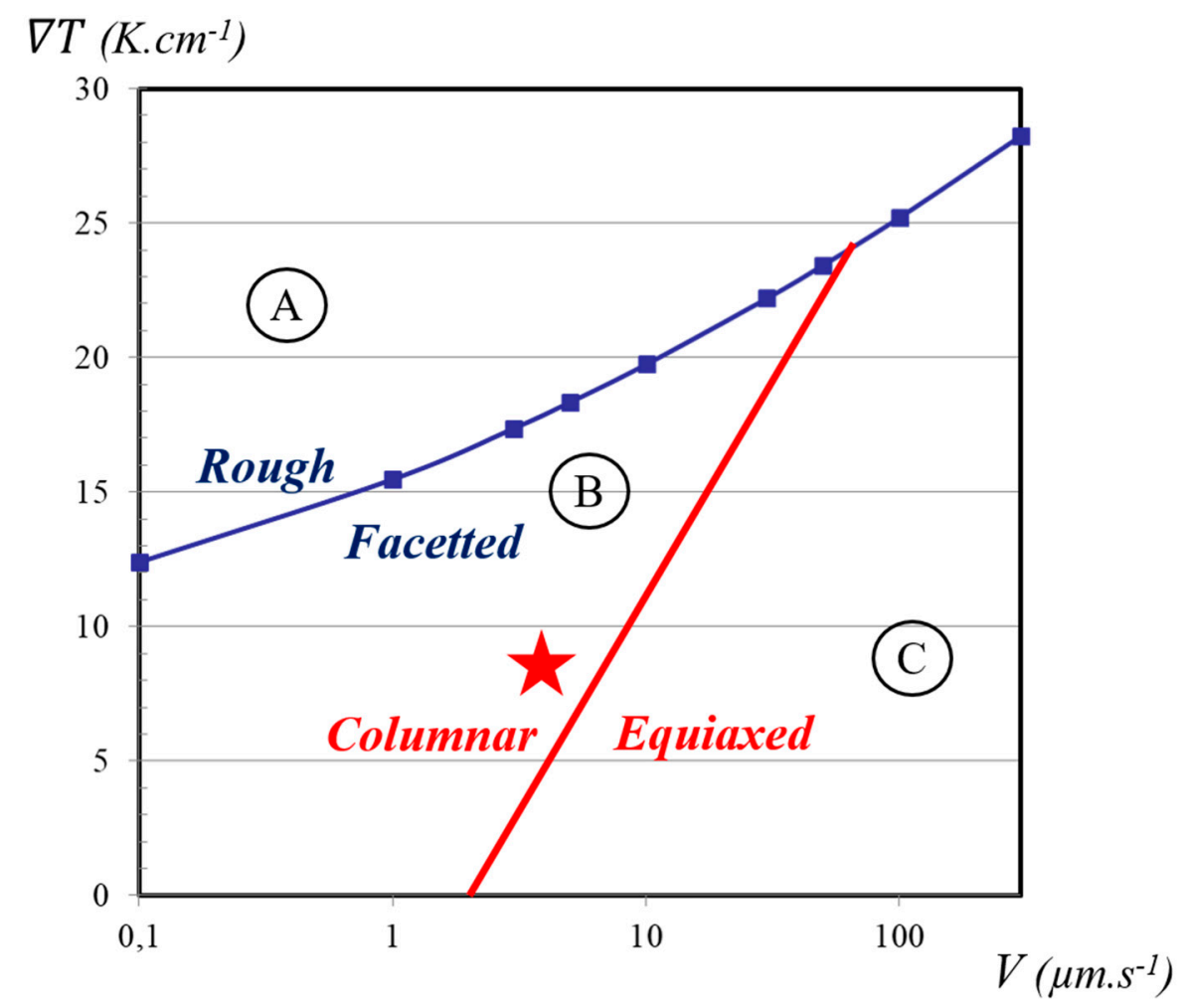

Figure 7. Diagram showing the various zones where typical grain structures appear in Photovoltaic Si ingots.

\section{Conclusions and Perspectives}

These two examples are limited to $V-\nabla T$ diagrams. However, there are many variations on this basis. For instance, we recently developed a $V-T_{\text {Die }}$ diagram in order to study the pulling of sapphire and eutectic oxide plates from a die, taking into account capillarity, hydrodynamics and heat transfer [18]. This shows that chart optimization can be used for many different growth techniques, provided convenient process parameters are chosen. 
On the one hand, the interest in this approach lies in its simplicity, requiring very little time and incurring virtually no cost. This is the case when the physical basis is already well known. Of course, things take much longer when they have to be first developed, as in the case of PV Si as described above, but this is more of an exception than the rule.

On the other hand, the accuracy of the results is limited, as rather crude approximations are performed in order to obtain analytical solutions to the physical problem. Often, more complex models can be found in the literature, but they generally require additional physical properties and then introducing uncertainties so that the benefits are doubtful in practical cases. Our experience is that simple, robust, analytical expressions provide a good order of magnitude for the expected solution, which can be used as the basis for further engineering developments.

A recurrent problem concerns the physical parameters of the material under study. In fact, there are only a few materials for which they are reasonably well known ( $\mathrm{Si}$, sapphire, typical binary semiconductors, etc.). In the other cases, only approximated values are available, most often by comparison with other materials of the same family. This is a further reason to use rather simple equations: the result accuracy is also limited by the available physical data.

As already pointed out, another limitation concerns the lack either of physical models or of their analytical solution. Sometimes, it is possible, when the time and manpower are available, to work out useful models, as shown in Part 4 above. However, many phenomena are still imperfectly understood, such as, for example, the behavior of point defects in binary semiconductors or in dielectric crystals, or the engulfment of bubbles and precipitates in almost all crystal growth processes, to list a few. Twinning is understood as the random nucleation of a disoriented seed on facets; however, there is no available quantitative description of the phenomenon. In our opinion, the development of physical understanding, associated models and corresponding analytical solutions should be a priority in the field of crystal growth process engineering.

Concerning the possible future developments of the chart technique, an obvious one is to follow the way paved in the field of metallurgy, i.e., the development of software, connected to an appropriate thermodynamic and material properties database, able to plot a variety of diagrams. This would take into account engineering requirements, in terms of crystal size, quality and process yield. Software-writing tools are, today, available and mature enough in order to make this objective attainable without the need to completely develop a full code. It could further be linked to classical process numerical simulation software, in order to provide much more accurate engineering solutions.

Such tools could also include models taking into account the evolution with time of the process parameters $\left(V, \nabla T, C_{L} \ldots\right)$ in the real equipment, to be used in direct online control.

Finally, it is important to stress, once again, the pedagogic usefulness of this approach that has been demonstrated through many years of teaching crystal growth engineering.

Funding: This research received no external funding.

Acknowledgments: This text is a written development of the first part of my plenary talk given at the 19th International Conference on Crystal Growth and Epitaxy held from 28 July to 2 August 2019 in Keystone, CO, USA. I am very grateful to the conference organizing and scientific committees for this very honoring invitation. I also warmly thank the Editors of this Special Issue of Crystals, for their invitation to provide a paper of my choice. The topic described in this paper has enjoyed great success for its pedagogic usefulness. I thank very much the Grenoble INP-Phelma Engineering School for the freedom and confidence they gave me in developing these lectures. I am also indebted to the successive student generations for their interest and questions concerning these lectures. Finally, I am pleased to thank my colleague Kader Zaidat for the numerous discussions concerning crystal growth process engineering in the lab, and for continuing teaching this matter to our students in the school.

Conflicts of Interest: The authors declare no conflict of interest. 


\section{Nomenclature}

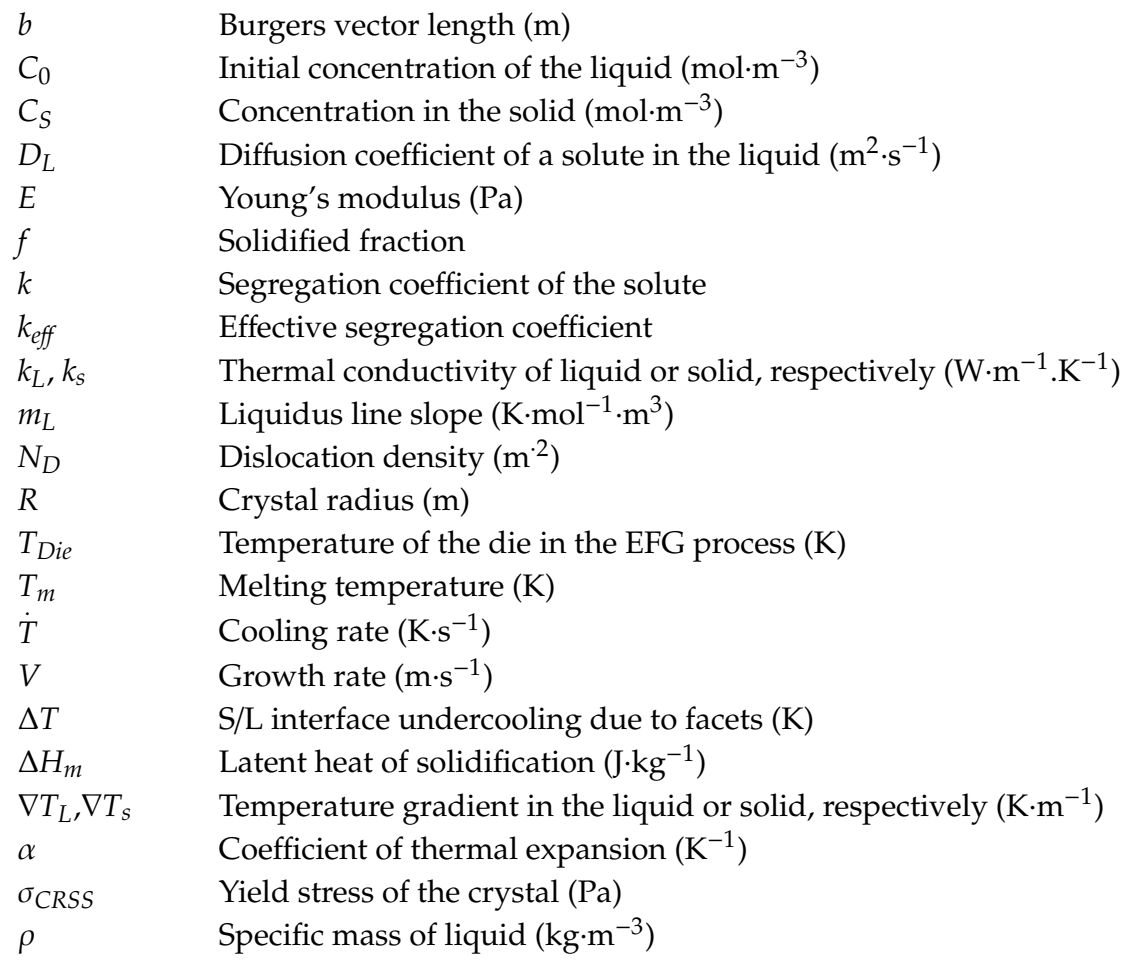

\section{References}

1. Trivedi, R.; Kurz, W. Microstructure and phase selection diagrams for directionally solidified binary alloys. In Proceedings of the Intelligence Processing Materials, Proceedings Symposium, Fall Meeting of the Minerals, Metals \& Materials Society, Indianapolis, Indiana, 2-5 October 1989; Wadley, H.N.G., Eckhart, E.W., Jr., Eds.; 1990; pp. 177-193.

2. Kurz, W. Solidification microstructure-processing maps: Theory and application. Adv. Eng. Mater. 2001, 3, 443-452. [CrossRef]

3. Stefan, J. Über die Theorie der Eisbildung. Ann. Phys. 1891, 278, 269-286. [CrossRef]

4. Duffar, T. Crystal Growth Processes Based on Capillarity: Czochralski, Floating Zone, Shaping and Crucible Techniques; John Wiley \& Sons: Chichester, UK, 2010; p. 566, ISBN 978-0-470-71244-3.

5. Tiller, W.A.; Jackson, K.A.; Rutter, J.W.; Chalmers, B. The redistribution of solute atoms during the solidification of metals. Acta Metall. 1953, 1, 428-437. [CrossRef]

6. Scheil, E. Bemerkungen zur Schichtkristallbildung. Z. Met. 1942, 34, 70-72.

7. Billig, E. Some defects in crystals grown from the melt-1. Defect caused by thermal stresses. Proc. R. Soc. Lond. 1956, 235, 37-55.

8. Indenbom, V.L. Stresses and dislocations in crystal growth. Izv. Nauk Akad. SSR Ser. Fiz. 1973, 37, $2258-2267$.

9. Drosd, R.; Washburn, J. Some observations on the amorphous to crystalline transformation in silicon. J. Appl. Phys. 1982, 53, 397-403. [CrossRef]

10. Duffar, T.; Nadri, A. On the twinning occurrence in bulk semiconductor crystal growth. Scr. Mater. 2010, 62, 955-960. [CrossRef]

11. Beatty, K.M.; Jackson, K.A. Monte Carlo modeling of silicon crystal growth. J. Cryst. Growth 2000, 211, 13-17. [CrossRef]

12. Duffar, T.; Nadri, A. The grain-grain-liquid triple phase line during solidification of multi-crystalline silicon. Comptes Rendus Phys. 2013, 14, 185-191. [CrossRef]

13. Mangelinck-Noël, N.; Duffar, T. Modelling of the transition from a planar faceted front to equiaxed growth: Application to photovoltaic polycrystalline silicon. J. Cryst. Growth 2008, 311, 20-25. [CrossRef]

14. Beaudhuin, M.; Duffar, T.; Lemiti, M.; Zaidat, K. One-dimensional model of the equiaxed grain formation in multi-crystalline silicon. J. Cryst. Growth 2011, 319, 106-113. [CrossRef] 
15. Beaudhuin, M.; Chichignoud, G.; Bertho, P.; Duffar, T.; Lemiti, M.; Zaidat, K. Carbon reaction with levitated silicon-Experimental and thermodynamic approaches. Mater. Chem. Phys. 2012, 133, 284-288. [CrossRef]

16. Lipton, J.; Glicksman, M.E.; Kurz, W. Equiaxed dendrite growth in alloys at small undercooling. Metall. Mater. Trans. A 1987, 18A, 341-345. [CrossRef]

17. Voigt, A.; Wolf, E.; Strunk, H.P. Grain orientation and grain boundaries in cast multicrystalline silicon. Mat. Sci. Eng. 1998, B54, 202-206.

18. Carroz, L.; Duffar, T. Tuning the sapphire EFG process to the growth of $\mathrm{Al}_{2} \mathrm{O}_{3} / \mathrm{YAG} / \mathrm{ZrO}_{2}: \mathrm{Y}$ eutectic. J. Cryst. Growth 2018, 489, 5-10. [CrossRef]

(C) 2020 by the author. Licensee MDPI, Basel, Switzerland. This article is an open access article distributed under the terms and conditions of the Creative Commons Attribution (CC BY) license (http://creativecommons.org/licenses/by/4.0/). 